\title{
Gravidez em Adolescentes com Dermatomiosite Juvenil (DMJ) $)^{(*)}$
}

\section{Presnancy in Adolescents with Juvenile Dermatomyositis (JDM)}

\author{
Clovis Artur Almeida Silva ${ }^{(1)}$, Marta Miranda Leal ${ }^{(2)}$, Marília Vieira Febrônio ${ }^{(3)}$, Georgiana Nogueira Leal ${ }^{(3)}$, \\ Adriana Maluf Elias Sallum ${ }^{(4)}$, Joelma Queiroz Andrade ${ }^{(5)}$, Marcelo Zugaib(6)
}

\section{RESUMO}

Com a melhora do prognóstico nos pacientes com dermatomiosite juvenil (DMJ), as crianças tornam-se adolescentes com possibilidade de início da vida sexual e risco de engravidar. No período entre 1983 e 2004, 4.638 pacientes foram seguidos em nosso serviço, entre os quais 117 (86/74\% do sexo feminino) apresentaram o diagnóstico de DMJ. Entre as pacientes com DMJ, três engravidaram durante o acompanhamento. A idade da primeira atividade sexual variou entre 15 (caso 1) e 14 anos (casos 2 e 3). As gestações, todas não planejadas, ocorreram aos 16 anos e 3 meses (caso 1), 15 anos e 4 meses (caso 2) e 14 anos e 7 meses (caso 3). A paciente 1 apresentava-se em atividade cutânea da DMJ no início da gravidez, mas evoluiu com completa remissão no $6^{\circ}$ mês da gestação; foram mantidos gamaglobulina endovenosa ( $2 \mathrm{~g} / \mathrm{kg} / \mathrm{mês})$ nos primeiros 5 meses e prednisona ( $5 \mathrm{mg} / \mathrm{dia})$ durante toda a gestação; o pré-natal foi sem intercorrências; o parto foi fórcipe; o recém-nascido (RN) nasceu sem intercorrências. As pacientes 2 e 3 estavam em remissão no início da gravidez e também não apresentaram intercorrências no período pré-natal. O parto da paciente 2 foi vaginal e o RN nasceu bem, de termo e adequado para idade gestacional. No caso 3, o parto foi cesariano por redução do líquido amniótico e o RN nasceu prematuro (36 semanas), mas bem e adequado para a idade gestacional. Em nenhuma das pacientes observou-se reativação da DMJ no período pós-natal. A atividade sexual precoce e o aumento da gravidez em adolescentes com DMJ são assuntos que devem ser considerados na rotina do atendimento desses pacientes.

Palavras-chave: dermatomiosite juvenil, adolescente, sexualidade, gravidez.

\begin{abstract}
The prognosis of patients with juvenile dermatomyositis (JDM) has improved, allowing adolescents and young adults to have an increased chance of sexual activities and pregnancy. From 1983 to 2004, 4,638 patients were followed at the Pediatric Rheumatology Unit of the Pediatric Department of FMUSP, of which 117 (86/74\% female) were diagnosed with JDM and 3 of them became pregnant during the follow-up. The first sexual intercourse occurred at 15 years old (case 1) and at 14 years old (cases 2 e 3). The pregnancies, all of them not planned, occurred at 16 years old and 3 months (case 1), 15 years old and 4 months (case 2) and 14 years old and 7 months (case 3). The disease of patient 1 was active when she became pregnant, but she was at remission by the end of the second trimester of gestation, she was given $2 \mathrm{~g} / \mathrm{kg} /$ month of intravenous immunoglobulin (during the first 5 months) and prednisone (during all the period); she had a full-term pregnancy with a normal birth. Patients 2 and 3 were on disease remission when they became pregnant and their gestations developed with no complications. Patient 2 had a full-term delivery; her newborn was adequate for gestational age and had no complications. Patient 3 had a Cesarean delivery due to low amniotic fluid; her newborn was premature (36 weeks) but adequate for gestational age and with no problems during neonatal period. None of the patients presented disease flare-up after delivery. Precocious sexual activity and pregnancy increase in adolescents with JDM should be considered in the routine clinical follow-up of these patients.
\end{abstract}

Keywords: juvenile dermatomyositis, adolescents, sexuality, pregnancy.

\footnotetext{
* Trabalho realizado nas Unidades de Reumatologia Pediátrica e de Adolescente do Departamento de Pediatria e Disciplina de Obstetrícia da Faculdade de Medicina da Universidade de São Paulo. Aprovado em 15/02/005. Aprovado, após revisão, em 26/03/2005.

1. Doutor em Medicina pela FMUSP. Responsável pela Unidade de Reumatologia Pediátrica do Departamento de Pediatria da FMUSP.

2. Mestre em Medicina pela FMUSP. Médica assistente da Unidade de Adolescente do Departamento de Pediatria da FMUSP.

3. Médica da Complementação Especializada da Unidade de Reumatologia Pediátrica do Departamento de Pediatria da FMUSP.

4. Mestre em Medicina pela FMUSP. Médica assistente da Unidade de Reumatologia Pediátrica do Departamento de Pediatria da FMUSP.

5. Doutor em Medicina pela FMUSP. Médica assistente do Setor de Colagenoses da Disciplina de Obstetrícia da FMUSP.

6. Professor titular da Disciplina de Obstetrícia da FMUSP.
}

Endereço para correspondência: Dr. Clovis Artur Almeida da Silva. R. Senador Cesar Lacerda Vergueiro, 494/82, Vila Madalena, CEP 05435-0 10. São Paulo, SP, Brasil. Tel. (11) 3069-8675; fax (11) 3069-8503; e-mail: clovisaas@icr.hcnet.usp.br 


\section{INTRODUÇÃO}

A dermatomiosite (DM) juvenil (DMJ) é a quarta doença crônica mais freqüente nos ambulatórios de Reumatologia Pediátrica, após a febre reumática, artrite idiopática juvenil e lúpus eritematoso sistêmico juvenil (LESJ). Seu diagnóstico precoce e a pronta instituição de terapêuticas medicamentosas e fisioterápicas propiciam, atualmente, uma melhora significativa na sobrevida e qualidade de $\operatorname{vida}^{(1-4)}$.

Com a melhora do prognóstico dessa doença, as crianças com DMJ evoluem além da infância e os reumatologistas pediátricos têm de lidar com questões próprias da adolescência agora vivida por seus pacientes, entre elas o início da sexualidade, risco de doenças sexualmente transmissíveis e gravidez ${ }^{(5)}$.

Impõe-se, então, dentro da especialidade, o desenvolvimento de programas rotineiros de educação sexual, principalmente porque as repercussões de uma gestação adquirem, na adolescente com DMJ, dimensão ainda maior, uma vez que na gravidez pode haver reativação da doença, com conseqüências maternas, fetais e no recém-nascido (RN).

A gravidez tem sido raramente descrita em pacientes adultas com miopatia inflamatória crônica: DM ou polimiosite $(\mathrm{PM})^{(6-9)}$. Silva et al. ${ }^{(6)}$ estudaram 43 casos de gestações em pacientes com DM ou PM, publicadas na literatura médica entre 1976 e 2001, entre as quais apenas duas (4\%) ocorreram em adolescentes com idade inferior a 16 anos: uma com $\mathrm{DMJ}^{(8)}$ e uma com $\mathrm{PM}^{(9)}$.

Este trabalho tem como objetivo relatar os casos de adolescentes com DMJ acompanhadas na Unidade de Reumatologia Pediátrica do Departamento de Pediatria da FMUSP, que engravidaram durante o seguimento ambulatorial. No período entre 1983 e 2004, essa Unidade acompanhou 4.638 pacientes, entre os quais 117 (2,5\%) apresentaram o diagnóstico de DMJ, segundo os critérios de Bohan e Peter ${ }^{(10)}$. Entre os pacientes com DMJ, 86 (74\%) eram do sexo feminino, três delas tendo evoluído para gravidez.

\section{RELATO DE CASOS}

\section{CASO 1}

Adolescente diagnosticada com DMJ aos 12 anos de idade pela presença de heliotropo, pápulas de Gottron em interfalangeanas proximais, fraqueza muscular proximal (força muscular grau IV nos membros superiores e grau III nos membros inferiores), elevação das enzimas musculares [creatinofosfoquinase (CK) $155 \mathrm{U} / 1$ (normal até 95U/1) e desidrogenase lática (DHL) 432 U/1 (normal até 240 U/1)] e biópsia muscular compatível com DMJ (presença de atrofia perifascicular e inflamação perivascular); apresentava, ainda, eritema malar e vasculites purpúricas nos membros inferiores. Iniciou-se tratamento com prednisona $(2 \mathrm{mg} / \mathrm{kg} / \mathrm{dia})$, havendo regressão da fraqueza muscular e normalização de suas enzimas musculares. Aos 12 anos e 8 meses houve piora das vasculites cutâneas e do eritema malar, sendo associada à prednisona, ciclosporina $(3 \mathrm{mg} / \mathrm{kg} / \mathrm{dia})$ e fotoprotetor solar (com fator 30). Aos 13 anos foi associada cloroquina $(5 \mathrm{mg} / \mathrm{kg} / \mathrm{dia})$ ao esquema anterior. Aos 14 anos, em conseqüência da intensificação da eritrodermia difusa no tronco e membros, heliotropo, pápulas de Gottron em interfalangeanas proximais e intenso eritema malar; substituiu-se a cloroquina por talidomida (100mg/dia) e aumentou-se a dose da ciclosporina $(5 \mathrm{mg} / \mathrm{kg} / \mathrm{dia})$. Aos 15 anos havia manutenção do quadro anterior, sendo suspensa a ciclosporina e talidomida, e introduzida pulsoterapia endovenosa com ciclofosfamida $\left(750 \mathrm{mg} / \mathrm{m}^{2} / \mathrm{mês}\right)$ por seis meses, com manutenção de $5 \mathrm{mg}$ de prednisona. Aos 16 anos houve recidiva das vasculites purpúricas nos membros inferiores e periungueais, sendo utilizada gamaglobulina endovenosa ( $2 \mathrm{~g} / \mathrm{kg} / \mathrm{mês})$, com manutenção de $5 \mathrm{mg}$ de prednisona. A idade de sua menarca foi de 14 anos. A sua primeira atividade sexual foi com 15 anos, com o seu primeiro namorado, que tinha 35 anos. Apesar de afirmar conhecer os métodos contraceptivos nessa ocasião, ela não os utilizou. Aos 16 anos e 3 meses a paciente estava grávida e em atividade cutânea da DMJ (vasculites periungueais, eritema malar e eritrodermia difusa). Abandonada por seu parceiro durante a gestação, a adolescente compartilhou essa gravidez apenas com seus pais, escondendo-a de seus amigos e outros familiares. Durante os cinco primeiros meses de gestação, manteve-se a gamaglobulina endovenosa $(2 \mathrm{~g} / \mathrm{kg} / \mathrm{mês})$; a prednisona $(5 \mathrm{mg})$ foi mantida por toda a gestação. Pela primeira vez desde o início da doença, a paciente evoluiu com completa remissão cutânea no sexto mês da gravidez e não apresentou intercorrências no acompanhamento pré-natal (sorologias para sífilis e HIV negativas). Aos 17 anos apresentou parto fórcipe, com apresentação cefálica, RN do sexo masculino, a termo, com idade gestacional de 37 semanas e adequado para a idade gestacional, peso de nascimento de $2.580 \mathrm{~g}$, Apgar 8, 9 e 10 (no primeiro, quinto e décimo minutos de vida), e icterícia fisiológica com necessidade de fototerapia por três dias. Houve rejeição do RN pela adolescente e por seus pais, que o encaminharam para adoção durante a internação no berçário. A adolescente evoluiu, no pós-parto, sem reativação da doença, com suspensão da prednisona aos 17 anos e 4 meses. Abandonou o seguimento aos 17 anos e 10 meses. 


\section{CASO 2}

Paciente com DMJ diagnosticada aos cinco anos de idade pela presença de heliotropo, fraqueza muscular proximal (força muscular grau III nos membros superiores e inferiores), edema nos antebraços, elevação das enzimas musculares [CK 441 U/1 (normal até 95U/1) e DHL 502 U/1 (normal até $240 \mathrm{U} / 1$ )] e biópsia muscular compatível com DMJ (presença de atrofia perifascicular e inflamação perivascular). Com a introdução de prednisona $(2 \mathrm{mg} / \mathrm{kg} / \mathrm{dia})$, houve regressão da fraqueza muscular e normalização de suas enzimas musculares. Aos seis anos, em uso de 7,5mg/ dia de prednisona, apresentou recidiva da doença com heliotropo, calcinoses (glúteos e coxas) e elevação das enzimas musculares [CK 98 U/1 (normal até 95 U/1) e DHL 318 U/1 (normal até $240 \mathrm{U} / \mathrm{l}$ )], sendo associada à prednisona, ciclosporina $(5 \mathrm{mg} / \mathrm{kg} / \mathrm{dia})$. Evoluiu com remissão da doença, sendo suspensa a ciclosporina aos oito anos e a prednisona aos 10 anos. Sua menarca ocorreu aos 13 anos e sua primeira atividade sexual aos 14 anos, com o seu primeiro namorado, de 17 anos. Apesar de afirmar conhecimento dos métodos contraceptivos nessa ocasião, ela não os utilizou. Aos 15 anos e 4 meses, em remissão da DMJ e sem uso de medicamentos, a paciente estava grávida. A gravidez, embora não planejada, foi compartilhada com seu parceiro e por ele aceita. A paciente evoluiu sem intercorrências no acompanhamento pré-natal (sorologias para sífilis, HIV, toxoplasmose, doença de Chagas e hepatites B e C negativas). A gestação foi a termo (idade gestacional de 38,5 semanas), o parto aos 16 anos foi vaginal com apresentação cefálica. O RN, do sexo masculino, foi a termo e adequado para a idade gestacional, com peso de nascimento de 3.180 gramas, Apgar 9, 10 e 10 (no primeiro, quinto e décimo minutos de vida) e recebeu alta do berçário normal com 24 horas de vida. No pós-parto evoluiu sem reativação da doença. Encontra-se, atualmente, com 19 anos e 10 meses e em remissão da DMJ.

\section{CASO 3}

Paciente com DMJ diagnosticada aos 9 anos e 4 meses de idade pela presença de heliotropo, pápulas de Gottron, fraqueza muscular proximal (força muscular grau III nos membros superiores e inferiores), elevação de enzimas musculares [DHL 1148 U/1(normal até 240 U/1) e aldolase 15,8mg/dl (normal até $7 \mathrm{mg} / \mathrm{dl}$ )] e biópsia muscular compatível com DMJ (presença de atrofia perifascicular, inflamação muscular e perivascular). Após introdução de prednisona $(2 \mathrm{mg} / \mathrm{kg} / \mathrm{dia})$ e difosfato de cloroquina $(5 \mathrm{mg} / \mathrm{kg} / \mathrm{dia})$, observou-se regressão da fraqueza muscular e das lesões cutâneas e normalização das enzimas musculares. Aos 12 anos apresentou pele acinzentada, sendo suspensa a cloroquina e mantida apenas com prednisona $(5 \mathrm{mg} / \mathrm{dia})$. A idade de sua menarca foi de 11 anos e a primeira atividade sexual ocorreu aos 14 anos, com o seu primeiro namorado, de 18 anos. Apesar de afirmar conhecimento sobre métodos contraceptivos por ocasião do início da vida sexual, ela não os utilizou. Aos 14 anos e 7 meses a paciente estava grávida e em remissão da DMJ; sendo mantida, durante a gestação, prednisona (5mg). Esta gravidez também não foi planejada, porém compartilhada com seu parceiro e por ele assumida. A paciente evoluiu sem intercorrências no pré-natal (sorologias para sífilis, HIV, toxoplasmose, doença de Chagas e hepatites B e C negativas). Aos 14 anos e 7 meses apresentou um parto prematuro (idade gestacional de 36 semanas), parto cesariano por redução do líquido amniótico, com apresentação cefálica. O RN, do sexo masculino, foi prematuro e adequado para a idade gestacional, peso de nascimento de 2.470 gramas, Apgar 9, 10 e 10 (no primeiro, quinto e décimo minutos de vida) e recebeu alta do berçário normal com 24 horas de vida. No pós-parto, a adolescente evoluiu sem reativação da doença e atualmente, com 16 anos, encontra-se em remissão.

\section{DISCUSSÃO}

As pacientes com DMJ sobrevivem à infância, tornam-se adolescentes com vida sexual, possibilidades de constituir família e questionamentos sobre a capacidade de gerar filhos. A escassez de publicações, na literatura médica, sobre o desenvolvimento e exercício da sexualidade em adolescentes com DMJ, assim como a ausência de estudos sobre a ocorrência de gravidez nessa população específica, estimulou a realização deste estudo.

A idade de início da atividade sexual das três pacientes aqui descritas variou de 13 a 15 anos, resultados compatíveis com os dados da população brasileira, cuja idade mediana da primeira relação sexual é de 15 anos; 42,5\% das adolescentes brasileiras iniciam sua vida sexual antes dos 18 anos de idade ${ }^{(11)}$. A redução da idade de início da vida sexual encontra-se entre os principais fatores responsáveis pelo aumento da gravidez na adolescência, juntamente com menarca mais precoce, influência da mídia e a fragilidade de um projeto pessoal de vida. O senso de indestrutibilidade ("comigo nada vai acontecer"), aliado à vivência temporal singular ("aqui e agora”) desse grupo etário são características próprias do desenvolvimento psicoemocional, que esclarecem a ocorrência de gravidez na adolescência, mesmo 
diante do conhecimento sobre e da possibilidade de acesso a métodos contraceptivos. A maioria das adolescentes que engravida conhece os métodos contraceptivos, porém não os utiliza ${ }^{(12)}$; as três pacientes com DMJ acompanhadas neste estudo também apresentaram esse perfil.

A gravidez na adolescência é um problema mundial. No Brasil, em 1997, mais de 700.000 partos (26\% do total) ocorreram em mulheres com menos de 20 anos de idade ${ }^{(13)}$. Mesmo representando um número pequeno diante do universo de adolescentes brasileiras que já engravidaram, o acompanhamento neste trabalho de três adolescentes grávidas da clientela adolescente feminina portadora de DMJ é preocupante e expressa os riscos a que estão sujeitas.

Em nossa Unidade de Reumatologia Pediátrica tem sido observado um aumento da freqüência de gravidez em adolescentes portadoras de doenças crônicas reumatológicas, refletindo o que acontece na sociedade como um todo. Um estudo realizado com 43 adolescentes com LESJ, seguidas em nossa Unidade e na Disciplina de Obstetrícia da FMUSP, no período entre 1999 e 2000, demonstrou a ocorrência de gravidez em 6 casos $(14 \%)^{(4)}$.

As taxas de fertilidade da DM ou PM podem estar diminuídas. A redução da função gonadal ovariana pode ocorrer pela própria atividade da doença crônica e/ou uso dos imussupressores $^{(15)}$. Por sua vez, King e Chow ${ }^{(7)}$ reportaram uma incidência de mulheres nulíparas de $12 \%$ em 78 pacientes com DM/PM; esses dados foram semelhantes aos encontrados na população geral. No presente estudo, apesar da atividade persistente da DMJ (caso 1) e uso prévio de imunossupressores (casos $1 \mathrm{e} 2$ ), as pacientes apresentaram fertilidade adequada. Estes achados também foram evidenciados por Silva et al. ${ }^{(16)} \mathrm{em}$ adolescentes com LESJ, sugerindo que nessa faixa etária a reserva folicular ovariana é normal, apesar da atividade da doença ou uso de medicamentos.

Em pacientes com miopatias inflamatórias (DM ou PM), a evolução da gravidez e atividade da doença ainda não está bem estabelecida, com poucos casos relatados. Silva et al. ${ }^{(6)}$ estudaram 43 casos de gestações de (DM ou PM no período entre 1976 e 2001. A doença pode estar presente antes do início da gravidez, ocorrer durante a gravidez ou no pósparto. Nesses 43 casos, 21 (49\%) pacientes apresentaram miopatia inflamatória antes de se tornarem grávidas, 15 (35\%) desenvolveram a doença durante a gravidez $(9$ com DM e 6 com PM) e 7 (16\%) no pós-parto. No presente estudo, as três pacientes com DMJ apresentavam a doença antes do início da gravidez.

Com relação à reativação da doença no momento da gestação ou no pós-parto, essa é uma situação excepcio- nalmente evidenciada. Habitualmente, a doença remite na gravidez com redução dos medicamentos ${ }^{(6)}$. Uma de nossas pacientes estava com atividade cutânea no início da gestação, controlada com prednisona e gamaglobulina endovenosa. Todos os três casos estavam em remissão no pósparto.

O prognóstico fetal está relacionado à atividade da doença materna. Abortamento, restrição do crescimento intra-uterino, prematuridade e morte do $\mathrm{RN}^{(6)}$ podem ocorrer em pacientes que têm a doença ativa no início da gestação, com pior prognóstico ${ }^{(17-19)}$. Na presente casuística, apenas um RN foi prematuro, sendo que todos evoluíram adequadamente no período neonatal imediato, sem maiores intercorrências.

$\mathrm{Na}$ literatura médica, a ocorrência de gravidez associada à DMJ foi descrita em apenas um caso. Pinheiro et al. ${ }^{(8)}$ descreveram o caso de uma adolescente brasileira, de 14 anos de idade, cuja DMJ iniciou durante o segundo trimestre da gravidez. A gestação evoluiu adequadamente com o tratamento (prednisona 2,0 mg/ $\mathrm{kg} / \mathrm{dia}$ ), necessitando de parto cesariano. O RN foi prematuro, sexo masculino, peso $2.050 \mathrm{~kg}$ e Apgar 1 e 7 no primeiro e quinto minutos, respectivamente.

O Colégio Americano de Reumatologia preconiza que os reumatologistas devam se responsabilizar pelos cuidados preventivos dos pacientes com doenças reumáticas crônicas, incluindo abordagem dos aspectos da sexualidade e contracepção ${ }^{(20)}$, lembrando-se de que a sexualidade é uma qualidade do ser humano e que a orientação sexual do adolescente não se baseia apenas no uso de métodos contraceptivos, mas no resgate do indivíduo, favorecendo o compromisso consigo mesmo (sexo responsável) e com o outro $^{(5)}$.

$\mathrm{Na}$ Unidade de Reumatologia Pediátrica, a orientação contraceptiva nas jovens com DMJ envolve o conceito de dupla proteção, ou seja, proteção contra gravidez e DST/ AIDS. Dessa forma, é dada ênfase ao uso de preservativo masculino ou feminino em todas as relações sexuais. $\mathrm{O}$ preservativo é um ótimo método contraceptivo e, diante de sua falha (ruptura, por exemplo), orienta-se o uso de contraceptivo de emergência (levonorgestrel, 1,5mg, dose única), o mais precoce possível. Não existem contra-indicações ao uso de contraceptivos hormonais combinados ou apenas de progestágeno de rotina para as pacientes portadoras de DMJ. Assim, em face da atividade sexual freqüente e/ou demanda da jovem, é possível prescrevê-los (sempre associados ao preservativo masculino $)^{(21)}$. Deve-se lembrar que a orientação anticoncepcional é um processo amplo e contínuo que se expande muito além do 
fornecimento de informações e conhecimentos sobre saúde reprodutiva; envolve o resgate do indivíduo, a promoção da auto-estima e a conscientização dos riscos vivenciados.

Os reumatologistas pediátricos estão acompanhando cada vez mais pacientes adolescentes com doenças crônicas, como a DMJ. O atendimento ao adolescente exige a capacidade de discutir com os jovens além dos aspectos da sexualidade e contracepção, riscos e momento ideal da gestação,

\section{REFERÊNCIAS}

1. Sogabe T, Silva CA, Kiss MH: Clinical and laboratorial characteristics of 50 children with Dermato/polymyositis. Rev Bras Reumatol 36: 351-9, 1996.

2. Sallum AM, Kiss MH, Sachetti S, et al: Juvenile dermatomyosistis. Clinical, laboratorial, histological, therapeutical and evolutive parameters of 35 patients. Arq Neuropsiquiatr 60: 889-99, 2002.

3. Nukumizu LK, Barros PC, Liphaus BL, Sallum AM, Silva CA: Sobreposição de dermatomiosite juvenil e lúpus eritematoso sistêmico. Relato de caso. Rev Bras Reumatol 42: 407-10, 2002.

4. Sallum AM, Marie SK, Wakamatsu A, et al: Immunohistochemical analysis of adhesion molecule expression on muscle biopsy specimens from patients with juvenile dermatomyositis. J Rheumatol 31: 801-7, 2004.

5. Silva CA, Leal MM, Campos LM, et al: Aspectos da sexualidade e gravidez de adolescentes e adultos jovens com lúpus eritematoso sistêmico juvenil (LES). Rev Bras Reumatol 41: 213-9, 2001.

6. Silva CA, Sultan SM, Isenberg DA: Pregnancy outcome in adult-onset idiopathic inflammatory myopathy. Rheumatology 42: 1168-72, 2003.

7. King CR, Chow S: Dermatomyositis and pregnancy. Obstet Gynecol 66: 589-92, 1985.

8. Pinheiro GR, Goldenberg J, Atra E, Pereira RB, Camano L, Schmidt B: Juvenile dermatomyositis and pregnancy: report and literature review. J Rheumatol 19: 1798-801, 1992.

9. Houck W, Melnyk C, Gast MJ: Polymyositis in pregnancy. A case report and literature review. J Reprod Med 32: 208-10, 1987.

10. Bohan A, Peter JB: Polymyositis and dermatomyositis. N Engl J Med 292: 344-7, 1975. prevenção das doenças sexualmente transmissíveis, teratogenicidade de algumas das drogas utilizadas para tratamento, assim como conscientização dos riscos da doença e dos medicamentos sobre a sua fertilidade.

O presente estudo evidencia a presença de atividade sexual precoce e gravidez na adolescência em pacientes com DMJ, o que impõe a discussão dos aspectos da sexualidade e contracepção rotineira.

11. Badiani R, Quental I, Santos EM: DST/AIDS e a pesquisa nacional sobre demografia e saúde: uma análise do nível de conhecimento e comportamento de vulnerabilização. Rio de Janeiro, BEMFAM/ DHS, 1997.

12. Saito MI: Sex education in school: preventing unwanted pregnancy in adolescents. Inter J Gynecol Obstetr 63: 157-60, 1998.

13. Serviço de Assistência à Saúde do Adolescente do Ministério da Saúde (SASAD): Prevenção intersetorial da gravidez na adolescência. Documento preliminar. São Paulo, Brasil, 1997.

14. Pinto e Silva JL, Sarmento RC: Gravidez. In: Adolescência e saúde - comissão de saúde do adolescente, 3.a ed, Secretaria de Estado da Saúde de São Paulo, 1999. p. 131-42.

15. Gutierrez G, Dagnino R, Mintz G: Polymyositis/dermatomyositis and pregnancy. Arthritis Rheum 27: 291-4, 1984.

16. Silva CA, Leal MM, Leone C, et al: Gonadal function in adolescents and young women with juvenile systemic lupus erythematosus. Lupus 11: 419-25, 2002.

17. Ishii N, Ono H, Kawaguchi T, Nakajima H: Dermatomyositis and pregnancy. Case report and review of the literature. Dermatologica 183: 146-9, 1991.

18. Solomon JE, D'Alton ME: Dermatomyositis in pregnancy. Curr Opin Obstet Gynecol 8: 83-6, 1996.

19. Ioannou Y, Sultan S, Isenberg DA: Myositis overlap syndromes. Curr Opin Rheumatol 11: 468-74, 1999.

20. American College of Rheumatology. The rheumatologist as principal care physician: In: ACR's Annual Scientific Meeting, Atlanta, 1998. p. 1-5.

21. World Health Organization. Department of Reproductive Health Research. Selected practice recommendations for contraceptive use. 2nd ed. WHO, Geneva, 2004. 